\title{
Fruit-induced Dormancy in Apple Seeds: Role of Water and Inhibitors
}

\author{
C.K. Wan ${ }^{1}$ and F.G. Dennis, Jr. \\ Department of Horticulture, Michigan State University, East Lansing MI 48824-1325 \\ Additional index words. Malus domestics, after-ripening, water content, volatiles, embryo, abscisic acid
}

\begin{abstract}
The roles of inhibitors in the seedcoat and locules and of seed water content in fruit-induced dormancy of apple (Malus domestics Borkh.) seeds were investigated. Seeds were after-ripened at 5C for various periods up to 12 weeks: 1) in the locules of intact fruits or half-fruits, 2) on wire mesh over moist filter paper, which simulated the locule in preventing leaching, or 3) on moist filter paper. The seeds were subsequently germinated in the locules of half-fruits, on screen, or on moist paper. In some experiments, the seeds were soaked in distilled water before or after after-ripening, or the embryos were excised before germination. The results indicate that fruit volatiles are unlikely to inhibit germination of seeds after-ripened in the fruit. Although limited water content reduced the ability of the seeds to germinate, it did not prevent after-ripening. An unidentified inhibitor(s) present in the locule and on the surface of the seed appears to be the major factor(s) preventing germination in the fruit, as seeds after-ripened on moist paper germinated well on paper or screen but poorly in the locule. The inhibitor(s) appears to be metabolized or to break down spontaneously when seeds are after-ripened on a screen, suggesting that the locule serves as a reservoir of the inhibitor as long as the seed remains in the fruit.
\end{abstract}

Apple seeds will not germinate until they have been subjected to a period of after-ripening under moist conditions at 0 to $10 \mathrm{C}$. However, seeds in fruit stored at such temperatures germinate very poorly on removal from the fruit, even after extended periods of storage (Bartlett, 1961; Pieniazek and Rudnicki, 1970; Visser, 1954). The low germination of such seeds has been attributed to 1) fruit-produced volatiles, including ethylene (Rudnicki and Pieniazek, 1973b); 2) insufficient moisture content for after-ripening (Bartlett, 1961); and 3) absence of free water to leach out seed inhibitors (Luckwill, 1952). In addition, a diffusible inhibitor in the endocarp could conceivably prevent germination.

The content of ABA, or an ABA-like inhibitor, declines rapidly during after-ripening of apple (Balboa-Zavala and Dennis, 1977; Rudnicki, 1969; Subbaiah, 1987) and other (Bonamy and Dennis, 1977; Lin and Bee, 1972; Liue and Crane, 1966) seeds that require moist chilling to terminate dormancy: Application of exogenous $\mathrm{ABA}$ inhibits germination of seeds and embryos in which dormancy has been broken (Kaminski, 1968; Pieniazek and Grochowska, 1967; Rudnicki and Pieniazek, 1973a). Thus, the breaking of dormancy has been ascribed to the decline in ABA content (Lipe and Crane, 1966; Rudnicki, 1969; Wareing and Saunders, 1971). However, Balboa-Zavala and Dennis (1977) found the decline in ABA to be independent of after-ripening temperature. Their data are questionable because of the methods used (Ozga and Dennis, 1991), but others have found either no decline in ABA (Ozga and Dennis, 1991) or a temperatureindependent decline (Subbaiah, 1987) in apple seeds. Thus, extractable ABA content does not appear to be the controlling factor in the breaking of seed dormancy. We now report on experiments that preceded these recent findings.

Our objectives were to determine the roles of water content vs. chemical inhibitors in the after-ripening and germination of apple seeds held in the fruits during after-ripening.

Received for publication 22 Feb. 1991. Accepted for publication 2 Dec. 1991. Research supported by the Michican Agricultural Experiment Station. The cost of publishing this paper was defrayed in part by the payment of page charges. Under postal regulations, this paper therefore must be hereby marked advertisement solely to indicate this fact.

'Current address: Agronomy-Horticulture Dept., Agricultural Univ., 43400 Serdang, Selangor, Malaysia.

\section{Materials and Methods}

Plant material. Mature 'Paulared' apple fruits were collected from the University Research Farm, East Lansing, Mich., in 1978 and 1979, along with 'Paulared' and 'Jonathan' apple fruits from a commercial orchard near Grand Rapids, Mich., in 1979. Some fruits had recently fallen from the trees and were collected from the ground. Seeds were removed either immediately or after storage of the fruits for varying periods at $5 \pm 1 \mathrm{C}$ and $85 \%$ relative humidity $(\mathrm{RH})$. None of the seeds was dried before use.

Generalprocedures. Experiments were designed to determine the influence of the various factors that might prevent seed germination in the fruit. Details are given below for each experiment. The effects of fruit-produced volatiles were tested by after-ripening seeds in petri dishes in closed pans with or without fruits. Water content was controlled by soaking seeds in water or holding them on a screen over moist paper. Inhibitor effects were evaluated by after-ripening and/or germinating seeds in the locule vs. on screen, or by soaking them (leaching) either before or after chilling. In several experiments, seeds were afterripened on one medium, then germinated on another, to determine whether after-ripening or germination was affected.

During after-ripening, seeds were kept 1) in the locules of intact or half-fruits, 2) on wire screen $(6.35-\mathrm{mm}$ mesh) over moist filter paper in petri dishes (paper). In some experiments, embryos were excised following after-ripening and their germination on paper or screen was compared with that of intact seeds. During after-ripening, fruits and petri dishes were held in darkness in a room maintained at $5 \pm 1 \mathrm{C}$ and a $\mathrm{RH}$ of $\approx 85 \%$. For germination, seeds or excised embryos (three replications of 20 per treatment) were kept in darkness for 10 days at $20 \pm$ 1C. Germination media are given for each experiment.

After-ripening location (Expt. 1). To distinguish between the effects of 1) inhibitors in the locules, 2) water content, and 3) presumed fruit volatiles, 'Paulared' apple seeds were after-ripened either in the locules of half-fruits or on screen in petri dishes. Half fruits and petri dishes were kept in covered plastic pans $(35 \times 20 \times 10 \mathrm{~cm})$ containing either petri dishes alone or petri dishes plus half-fruits. Half of the seeds in each treat-

Abbreviation: ABA, abscisic acid. 
ment were presoaked in distilled water for 24 or $48 \mathrm{~h}$ before after-ripening, the remainder was not. Samples were tested for germination on the same medium used for after-ripening (locules or screen after 3,6,9, and 12 weeks at $5 \mathrm{C}$ ). The experiment was repeated in 1979. To assure that moisture was not limiting germination, small droplets of Water were applied weekly to seed held on screen during germination.

After-ripening method (Expt. 2). 'Paulared' apple seeds were after-ripened 1) in intact fruit, 2) on screen, or 3) on paper. Samples were removed at 3-week intervals, and both seeds and excised embryos were germinated (on paper and screen). To determine seed water content (percent initial fresh weight), the seeds were blotted on paper towels, weighed, kept at $75 \mathrm{C}$ for $72 \mathrm{~h}$, then reweighed.

After-ripening and germination location (Expt. 3). 'Paulared' seeds were after-ripened for 12 weeks in intact fruits or in petri dishes on screen or paper. Half the seeds in each treatment were soaked for $24 \mathrm{~h}$ at $20 \pm 1 \mathrm{C}$, and samples were germinated in the locules of half-fruits or in petri dishes on screen or paper.

Blocking of inhibitors (Expt. 4). 'Jonathan' apple seeds afterripened on paper for 12 weeks were germinated on the endocarp of fruit sections placed horizontally on paper. Seeds were placed: 1) directly on the endocarp, 2) on moist paper in contact with the endocarp, 3) on moist paper backed by aluminum foil in contact with the endocarp, 4) on moist paper in a petri dish, or 5) on screen in a petri dish.

Soaking, seedcoat removal, and ABA (Expt. 5). 'Jonathan' fruits were stored at $5 \pm 1 \mathrm{C}$ for 12 weeks. The seeds were removed and soaked in distilled water for 0 to $72 \mathrm{~h}$ with or without the addition of ABA (10 ppm). The seeds were germinated on screen with the seedcoat intact, removed over the radicle end, or entirely removed (excised embryos).

Germination tests. Seeds or embryos were kept for 10 days at $20 \pm 1 \mathrm{C}$ on paper, screen, or the locule, unless otherwise stated. RH was maintained at $\approx 100 \%$.Seeds with visible radicle protrusion and embryos whose radicles showed geotropic curvature were considered germinated.

Statistical analysis. For each period of after-ripening and each seed covering (intact seed vs. embryo), the data were analyzed separately by analysis of variance; percent germination was converted to arcsin $\sqrt{\%}, \quad$ unless otherwise noted. Means were separated by LSD or Duncan's multiple range test.

\section{Results}

Experiment 1. All seeds left in the locules failed to germinate in 1978 and 1979 whether they had been soaked following afterripening or not (data not shown). Soaking in water following after-ripening stimulated germination of seeds after-ripened on screen (Table 1), except for the 12-week treatment in 1979. Germination was significantly delayed in the presence of halffruits, except after 12 weeks in 1979, suggesting some inhibition by volatiles.

Experiment 2. Seed water content (47\% of the fresh weight on removal from the fruit) remained unchanged during afterripening in the fruit or on screen, but rose to $57 \%$ to $58 \%$ on paper within 3 weeks, then stabilized. All interactions between after-ripening and germination medium were significant at $P<$ 0.001 . After-ripening seeds in the fruit strongly inhibited their subsequent germination in comparison with after-ripening on screen or on paper. No germination occurred when seeds were kept on screen after removal from the fruits (Fig. $1 \mathrm{~A}$ and C). In 1978, after-ripening on paper was more effective than after-
Table 1. Main effects of soaking seeds in water for $24 \mathrm{~h}$ before afterripening and of presence of half-fruits during after-ripening and germination on germination of 'Paulared' apple seeds. Seeds after-ripened at $5 \mathrm{C}$ for 9 or 12 weeks, then geminated at 20C (Expt. 1).

\begin{tabular}{|c|c|c|c|}
\hline \multirow[b]{3}{*}{ Treatment ( $5 \mathrm{C}$ for wk) } & \multicolumn{3}{|c|}{ Seed germination $(\%)$} \\
\hline & \multirow{2}{*}{$\frac{1978}{12 \mathrm{wk}}$} & \multicolumn{2}{|c|}{1979} \\
\hline & & $9 \mathrm{wk}$ & $12 \mathrm{wk}$ \\
\hline Not soaked & 29 & 8 & 75 \\
\hline Soaked & $88^{* * z}$ & $36^{* *}$ & 70 NS \\
\hline Without half-fruits & 66 & 29 & 70 \\
\hline With half-fruits & $51^{* *}$ & $16^{*}$ & $75 \mathrm{NS}$ \\
\hline
\end{tabular}

${ }^{2}$ Not significant (NS) or significantly different from respective controls (without half-fruits or not soaked) held at $5 \mathrm{C}$ for the same period of time at $P=0.05\left(^{*}\right)$ or $0.01\left({ }^{* *}\right)$ by $F$ test after conversion of percent germination to $\arcsin \sqrt{\%}$.

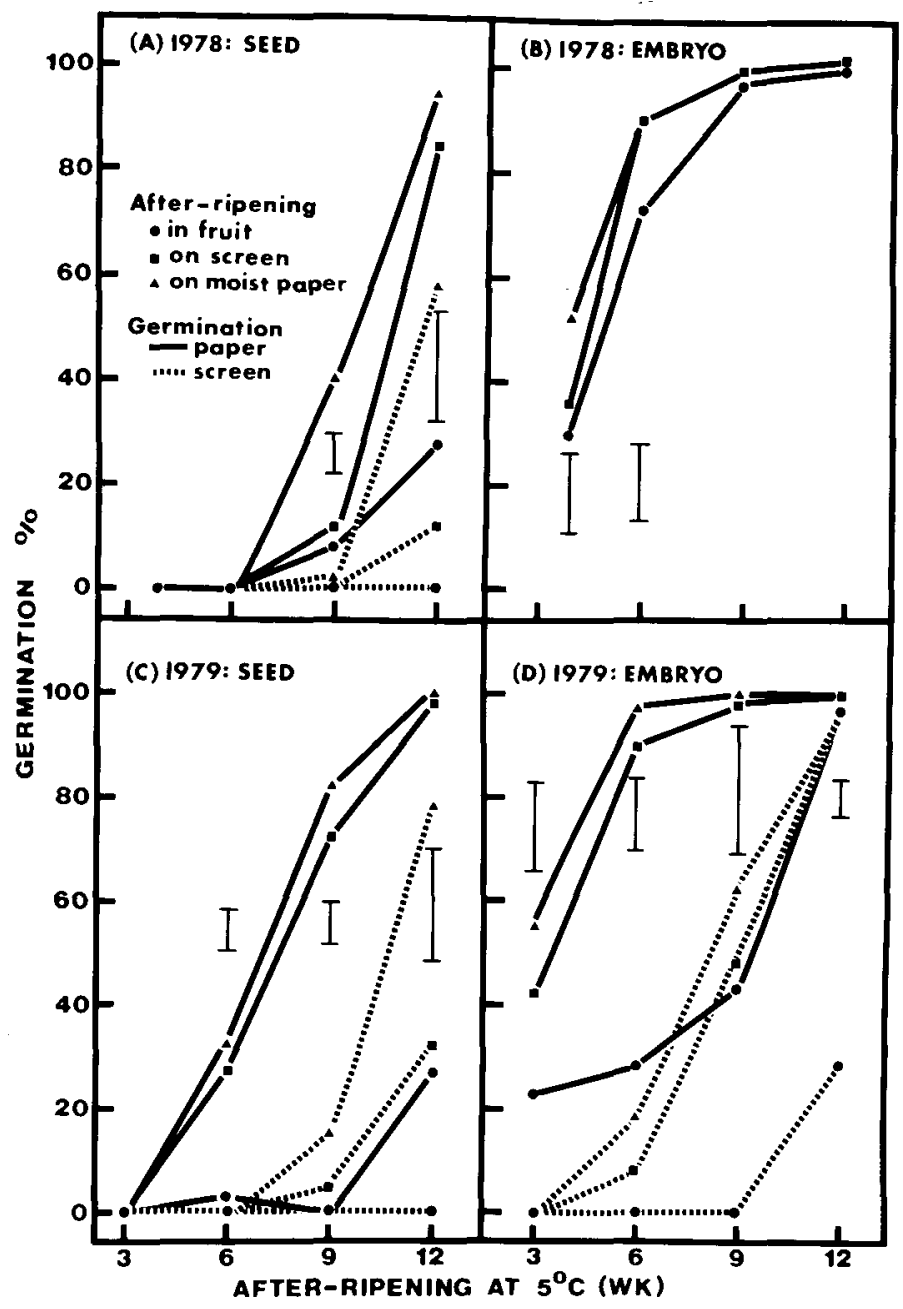

Fig. 1. Effects of method and time of after-ripening at 5C, and of germination medium, on germination at $20 \mathrm{C}$ of 'Paulared' apple seeds and excised embryos (Expt. 2). Vertical bars indicate LSDS at $P<0.01$ for each time of after-ripening.

ripening on screen, but in 1979, differences were nonsignificant unless the seeds were germinated on screen.

In 1978, all embryos were germinated on paper. Germination of embryos from seeds after-ripened on screen or in the fruit was delayed relative to those after-ripened on paper (Fig. 1B). There were not significant differences after 9 weeks of afterripening. In 1979, germination percentage was similar in em- 
bryos excised from seeds after-ripened on paper, in fruits, or on screen regardless of germination medium (Fig. ID); however, keeping seeds in the fruit inhibited germination after 3 to 9 weeks of after-ripening. Germination on screen was markedly delayed relative to that on paper, regardless of after-ripening medium.

Experiment 3. Seeds after-ripened and/or germinated" in the locules consistently germinated poorly in comparison with those held in petri dishes, and no germination occurred in seeds that were after-ripened and germinated in the locules (Table 2). Afterripening on screen was less effective than after-ripening on paper unless the seeds were soaked after treatment or germinated on paper. Soaking following after-ripening consistently stimulated germination on screen, but response on paper and in the locule varied, depending on after-ripening medium.

Experiment 4 . When fully after-ripened seeds were placed on the endocarp of fruit sections, only $38 \%$ germinated in comparison with $98 \%$ on screen and $100 \%$ on paper. When moist paper was inserted between the endocarp and the seed, germination increased to $88 \%$ - significantly less $(\mathrm{P}<0.05)$ than the $97 \%$ observed on paper alone or on paper isolated from the endocarp by aluminum foil. This difference indicates a small but definite inhibitory effect of material diffusing from the endocarp.

Experiment 5. Response to imbibition varied depending on

Table 2. Effect of after-ripening and germination media and soaking in water following after-ripening on percentage of germination of 'Paulared' apple seeds. Seeds after-ripened for 12 weeks at $5 \mathrm{C}$, then germinated at 20C (Expt. 3).

\begin{tabular}{lcccr}
\hline \hline \multirow{2}{*}{$\begin{array}{c}\text { After-ripening } \\
\text { medium }\end{array}$} & Soaked & Locule & Paper & Screen \\
\cline { 3 - 5 } Locule & - & $0 \mathrm{~d}^{\mathbf{z}}$ & $26 \mathrm{c}$ & $0 \mathrm{~d}$ \\
& + & $0 \mathrm{~d}$ & $42 \mathrm{c}$ & $26 \mathrm{c}$ \\
Paper & - & $38 \mathrm{c}$ & $100 \mathrm{a}$ & $78 \mathrm{~b}$ \\
- & + & $40 \mathrm{c}$ & $98 \mathrm{a}$ & $98 \mathrm{a}$ \\
Screen & - & $0 \mathrm{~d}$ & $98 \mathrm{a}$ & $32 \mathrm{c}$ \\
& + & $40 \mathrm{c}$ & $98 \mathrm{a}$ & $98 \mathrm{a}$ \\
\hline
\end{tabular}

${ }^{7}$ Mean separation among all treatments (rows and columns) by Duncan's multiple range test $P=0.01$ after transformation to $\arcsin \sqrt{\%}$. All interactions significant at $P<0.01$.

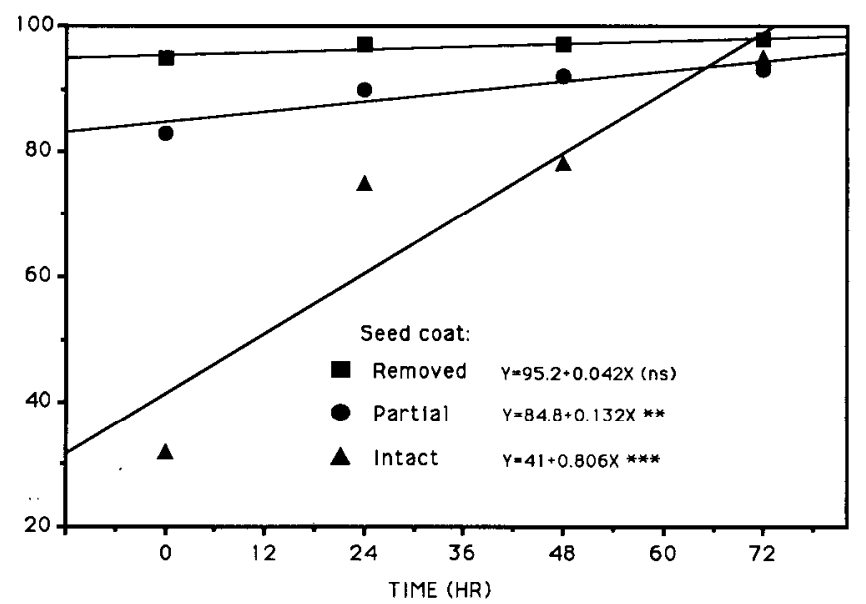

Fig. 2. Effect of soaking time in water and subsequent seedcoat removal on germination of apple seeds, cultivar Jonathan, after-ripened in the fruit for 12 weeks at 5C, then germinated on screen (Expt. 5). Partial $=$ seedcoat removed over radicle. Linear regression significant at $P<0.01(* *)$ or $P<0.001(* * *)$. seedcoat treatment (Fig. 2). Germination of intact seeds and partially excised embryos increased with time of soaking in water, whereas that of embryos (seedcoat removed) was $>95 \%$ regardless of soaking time. Removal of the seedcoat over the radicle was nearly as effective as complete removal in stimulating germination of embryos from nonimbibed seeds (difference not significant by analysis of variance). Inclusion of ABA $(10 \mathrm{ppm})$ in the water used for soaking did not significantly affect germination of seeds or embryos (data not shown). Overall means for $\mathrm{ABA}$ at 0 and $10 \mathrm{ppm}$ were $91 \%$ and $88 \%$, respectively.

Percent germination varied considerably among experiments. For example, the data in Fig. 1 (Expt. 2) indicate 'Paulared' seeds collected in 1978 were less responsive to chilling than those collected in 1979. In contrast, the data in Table 1 (Expt. 1) indicate a greater response to 12 weeks of chilling in seeds collected in 1978. Time of collection and length of storage may affect germination; therefore, comparisons are valid only within seed lots and experiments.

\section{Discussion}

Four hypotheses are proposed to explain the inhibition of germination of apple seeds after-ripened in the fruit: 1) inhibition by volatiles produced by the fruit, 2) insufficient water content to permit after-ripening, 3) insufficient contact with water to leach inhibitors from the seed, and 4) inhibitor(s) located in the locule.

Volatiles. When seeds were after-ripened on screen, the presence of half-fruits had only a small effect on their subsequent germination (Table 1), whereas seeds in the locules of halffruits consistently germinated poorly. These data, together with experiments on the effects of ethylene on apple seed dormancy (Ozga and Dennis, 1989; Wan, 1980), make the role of volatiles questionable at best.

Insufficient water for after-ripening. Williams (1985) reported that water contents $\geq 8 \%$ to $12 \%$ of dry weight were necessary for chilling to be effective in breaking apple seed dormancy. In our experiments, seeds after-ripened on screen contained the same amount of water $(47 \%$ of fresh weight $=$ $89 \%$ of dry weight) as those kept in the fruit (Expt. 2), yet the former germinated better following after-ripening (Expt. 2, Fig. 1). Seeds after-ripened on screen germinated as well as those after-ripened on paper, provided they were soaked before germination (Table 2). Thus, after-ripening had occurred, but lack of water limited germination.

Insufficient contact with water to leach inhibitors from the seed. If leaching were essential for removing an inhibitor, seeds after-ripened on screen should germinate no better than those held in the fruit. However, screen was a consistently better medium than the locule (Table 2, Fig. 1). Although soaking following after-ripening does indeed stimulate germination of seeds after-ripened on screen (Tables 1 and 2), this is probably an effect of water content, rather than of leaching. For example, nonsoaked seeds that were after-ripened on screen, then germinated on paper, germinated better than those that were afterripened on paper, then germinated on screen (Fig. 1, Table 2), yet the former were exposed to the moist paper for a much shorter time than were the latter (10 days vs. 12 weeks).

An inhibitor in the locule. The presence of an inhibitor in the locule appears to be the most plausible explanation for poor germination. If the locule were "neutral," it should be similar to screen in affecting germination. Yet seeds after-ripened in thelocule failed to germinate there, whether they were soaked 
or not (Table 2), and seeds that were after-ripened on either paper or screen germinated poorly in the locule despite soaking (Table 2). When moist paper or aluminum foil was used to line the locule, germination was greatly improved (Expt. 4). Measurement of endogenous ABA in locule extracts by electroncapture gas chromatography was attempted (data not shown). The technique was inaccurate and ABA content was overestimated (maximum $2.5 \mu \mathrm{g} \cdot \mathrm{g}^{-1}$ fresh weight $=2.5 \mathrm{ppm}$ ). However, even this concentration is below that required [20 ppm $( \pm) \mathrm{ABA}=10 \mathrm{ppm}(+) \mathrm{ABA}]$ for $50 \%$ inhibition of apple seed germination (Rudnicki and Pieniazek, 1973a, 1973b), suggesting that $\mathrm{ABA}$ is not the inhibitor. (Note that $10 \mathrm{ppm}$ used in Expt. 5 failed to inhibit germination).

The germination capacity of seeds after-ripened on screen increased more rapidly than that of seeds kept in the locules (Expt. 1; Tables 1 and 2; Fig. 1). This result suggests that the inhibitor(s) is metabolized, or breaks down spontaneously, when seeds are separated from the fruit, but either remains unaltered or is renewed when the seed remains in the locule.

The inhibitor remains to be identified. It could conceivably block the biosynthesis of hormones such as gibberellins and cytokinins in the embryo, or it may be nonspecific in effect, high concentrations being sufficient to inhibit all growth.

\section{Literature Cited}

Balboa-Zavala, O. and F.G. Dennis, Jr. 1977. Abscisic acid and apple seed dormancy. J. Amer. Soc. Hort. Sci. 102:633-637.

Bartlett, C.E.C. 1961. The after-ripening of apple seeds in the fruit during cold storage. Annu. Rpt. Long Ashton Agr. Hort. Res. Sta. 1961:66-67.

Bonamy, P.A. and F.G. Dennis, Jr. 1977. Abscisic acid levels in seeds of peach. II. Effects of stratification temperature. J. Amer. Soc. Hort. Sci. 102:26-28.

Kaminski, W. 1968. Inhibitory effect of apple juice on the germination of apple and cherry seeds and the growth of apple seedlings. Acts Societatis Botanicorum Poloniae 37:173-178.

Lin,.C.F. and A.A. Bee. 1972. Effects of some endogenous and ex- ogenous growth regulators on plum seed dormancy. J. Amer. Soc. Hort. Sci, 97:41-44.

Lipe, W. and J.C. Crane. 1966. Dormancy regulation in peach seeds. Science 153:541-542.

Luckwill, L,C. 1952. Growth-inhibiting and growth-promoting substances in relation to the dormancy and after-ripening of apple seeds. J. Hort, Sci. 27:53-65.

Ozga, J. and F.G. Dennis, Jr. 1989. Is ethylene required for apple seed or embryo germination? HortScience 24:331-333.

Ozga, J.A. and F.G. Dennis, Jr. 1991. The role of abscisic acid in heat stress induced secondary dormancy in apple seeds. HortScience 26:175-177.

Pieniazek, J. and M.J. Grochowska. 1967. The role of the natural growth inhibitor (abscisin II) in apple seed germination and the changes in the content of phenolic substances during stratification. Acts Societatis Botanicorum Poloniae 36:579-587.

Pieniazek, J; and R. Rudnicki. 1970. The inhibitory effect of endogenous abscisic acid (ABA) on after-ripening of apple seeds in the fruit. Bul. Academie Polonaise Sci. 27:707-711.

Rudnicki, R. 1969. Studies on abscisic acid in apple seeds. Planta 86:63-68.

Rudnicki, R. and J. Pieniazek. 1973a. The effect of abscisic acid on stratification of apple seeds. Bul. Academie Polonaise Sci. 21:149154.

Rudnicki, R. and J. Pieniazek. 1973b. Apple fruit volatiles and inhibitors of apple seed germination. Bul Academie Polonaise Sci. 21:827829.

Subbaiah, T. 1987. Abscisic acid relationships in apple seed dormancy. PhD Diss., Cornell Univ., Ithaca, N.Y.

Visser, T. 1954. After-ripening and germination of apple seeds in relation to the seed coats. Proc. Koninklijke Nederlandse Akademie van Wetenschappen C57:175-185.

Wan, C.K. 1980. The role of ethylene in seed dormancy with particular reference to apple (Malus domestics Borkh H.) seeds. Pertanika 3:78-81.

Wareing, P.F. and P.F. Saunders. 1971. Hormones and dormancy. Annu. Rev. Plant Physiol. 22:261-288.

Williams, K.M. 1985. ATPase activity and water uptake in apple seeds during stratification. PhD Diss., Cornell Univ., Ithaca, N.Y. 